\title{
Bioequivalence of the Emtricitabine/Rilpivirine/Tenofovir Disoproxil Fumarate Single Tablet Regimen
}

\author{
A Mathias*, M Menning, L Wiser, X Wei, A Dave, S Chuck and BP Kearney
}

Gilead Sciences, Inc., 333 Lakeside Drive, Foster City, CA 94404, USA

\begin{abstract}
Emtricitabine/rilpivirine/tenofovir disoproxil fumarate (FTC/RPV/TDF) is a next-generation, once-daily complete antiretroviral single-tablet regimen for the treatment of HIV-1 infection in adults. This study evaluated the pharmacokinetics and bioequivalence of 2 distinct investigational coformulations of the FTC/RPV/TDF single-tablet regimen (containing emtricitabine $200 \mathrm{mg}$, rilpivirine $25 \mathrm{mg}$, and tenofovir disoproxil fumarate $300 \mathrm{mg}$ ) compared with the concurrent administration of a 200-mg strength capsule of FTC, a 25-mg strength tablet of RPV, and a 300-mg strength tablet of tenofovir disoproxil fumarate in healthy subjects. Thirty-six subjects were randomized in a single-dose, open-label, 3-way crossover study design; 34 subjects completed all study treatments. Serial blood samples were obtained over 192 hours following oral administration of each treatment and pharmacokinetic parameters calculated. Formulation bioequivalence was assessed by $90 \%$ confidence intervals $(\mathrm{Cl})$ for the ratio of geometric least square means (GMR) for $\mathrm{C}_{\max }, \mathrm{AUC}_{0 \text {-last }}$ and $\mathrm{AUC}_{\text {inf }}$ for each drug of the FTC/RPV/TDF single-tablet regimen versus the individual components. Emtricitabine, rilpivirine, and tenofovir disoproxil fumarate administered under fed conditions (standardized meal of $\sim 400 \mathrm{kcal}$ ) as individual drugs given concurrently or as a fixed-dose combination tablet were generally well tolerated. Of the 2 single-tablet formulations that were tested, a single coformulation (Test Formulation 1) demonstrated bioequivalence to the reference formulation, with $90 \%$ confidence intervals for the ratio of the geometric least-squares means contained within the bounds of $80 \%$ to $125 \%$ for the $A U C_{\text {inf }}, A C_{0-\text { last }}$, and $C_{\text {max }}$ values of emtricitabine, rilpivirine, and tenofovir, and was carried forward as the commercial formulation. This tablet is a next-generation, once-daily single-tablet antiretroviral regimen for the treatment of HIV-1 infection and offers an attractive treatment option to efavirenz-containing regimens.
\end{abstract}

Keywords: Bioequivalence; Emtricitabine; Rilpivirine; Tenofovir disoproxil fumarate; Single-tablet regimen; HIV-1

\section{Introduction}

Clinical studies have demonstrated high levels of adherence and treatment satisfaction with simple, once-daily highly active antiretroviral therapy (HAART) regimens, resulting in durable suppression of HIV-1 RNA [1,2]. The availability of the efavirenz/ emtricitabine/tenofovir disoproxil fumarate single-tablet regimen as well as other combination tablets has enabled simplification of HIV treatment. Before the recent approval of the emtricitabine/rilpivirine/ tenofovir disoproxil fumarate (FTC/RPV/TDF) single-tablet regimen, efavirenz $600 \mathrm{mg} / \mathrm{em}$ tricitabine $200 \mathrm{mg} /$ tenofovir disoproxil fumarate $300 \mathrm{mg}$ (EFV/FTC/TDF) was the only fixed-dose, triple combination single-tablet regimen for the treatment of HIV. Thus, there remains a need for new fixed-dose single-tablet regimens composed of potent agents exhibiting favorable tolerability, minimal short- and long-term toxicity, and convenient dosing to maximize patient adherence.

Current guidelines for the treatment of antiretroviral (ARV) treatment-naive HIV-1 infected patients recommend 2 nucleos $(\mathrm{t})$ ide reverse transcriptase inhibitors (NRTIs/N[t]RTIs) and a nonnucleoside reverse transcriptase inhibitor (NNRTI) as a preferred initial therapy [3-6]. The use of the NNRTIs nevirapine, delavirdine, etravirine, and efavirenz are limited by cross resistance, frequent dosing, and/or safety issues that may include hepatotoxicity, rash, central nervous system symptoms, and teratogenicity. An alternative next-generation NNRTI, rilpivirine, a diarylpyrimidine (DAPY) derivative, has recently been approved [7]. Data from Phase 2 and 3 studies indicated that RPV $25 \mathrm{mg}$ had the potential to combine the convenience of once-daily dosing with potent antiviral efficacy similar to efavirenz, with an improved safety profile over efavirenz with respect to central nervous system adverse events, lipid abnormalities, incidence of rash, and lack of teratogenicity
[7-12]. Forty-eight week efficacy data and safety data from the Phase 3 clinical trials ECHO (NCT00540449) and THRIVE (NCT00543725) in treatment-naïve HIV-1 infected subjects demonstrated that rilpivirine $25 \mathrm{mg}$ administered in combination with NRTI/NtRTI backbone agents, including the preferred emtricitabine 200-mg and tenofovir disoproxil fumarate- $300 \mathrm{mg}$ backbone, was noninferior to efavirenz $600 \mathrm{mg}$ administered in combination with the same NRTI/NtRTI backbone agents $[3,4,9,10]$.

Rilpivirine has been coformulated with the standard-of-care NRTI/ NtRTI backbone FTC/TDF into a single-tablet regimen (FTC/RPV/ TDF). The FTC/RPV/TDF single-tablet regimen was approved for use by the FDA in August 2011 as a complete regimen for the treatment of HIV-1 infection in treatment-naïve adults [12] and it could offer an alternative single-tablet regimen option to patients who wish to simplify their treatment regimen and avoid the complicated dosing restrictions and timing imposed by multidrug regimens.

The present study was conducted during the development of the FTC/RPV/TDF single-tablet regimen to evaluate the bioequivalence of coformulations of FTC/RPV/TDF (containing $200 \mathrm{mg}$ emtricitabine,

*Corresponding author: Anita Mathias, Gilead Sciences, Inc., 333 Lakeside Drive, Foster City, CA 94404, USA, Tel: (650) 522-3000; Fax: (650) 522-5801; E-mail: anita.mathias@gilead.com

Received September 10, 2012; Accepted September 20, 2012; Published September 25, 2012

Citation: Mathias A, Menning M, Wiser L, Wei X, Dave A, et al. (2012) Bioequivalence of the Emtricitabine/Rilpivirine/Tenofovir Disoproxil Fumarate Single Tablet Regimen. J Bioequiv Availab 4: 100-0105. doi:10.4172/jbb.1000121

Copyright: (C) 2012 Mathias A, et al. This is an open-access article distributed under the terms of the Creative Commons Attribution License, which permits unrestricted use, distribution, and reproduction in any medium, provided the original author and source are credited. 
$25 \mathrm{mg}$ rilpivirine, and $300 \mathrm{mg}$ tenofovir disoproxil fumarate) compared with the concurrent administration of a $200-\mathrm{mg}$ strength capsule of emtricitabine, a $25-\mathrm{mg}$ strength tablet of rilpivirine, and a $300-\mathrm{mg}$ strength tablet of tenofovir disoproxil fumarate under fed conditions. Assessment of the bioequivalence under fed conditions was considered appropriate as rilpivirine exhibits a positive food effect; exposure to rilpivirine is reduced $\sim 40 \%$ when dosed in a fasted state compared to dosing following a normal- or high-caloric meal. Administration once daily with a meal is recommended in the product labeling for both rilpivirine and the single-tablet regimen of FTC/RPV/TDF $[7,12]$.

\section{Methods}

\section{Study population}

A total of 36 healthy male and nonpregnant, nonlactating female subjects, between 18 and 45 years of age (inclusive), with a body mass index (BMI) between 19 and 30 (inclusive), no significant medical history, and a creatinine clearance $\geq 80 \mathrm{~mL} /$ minute (using the Cockcroft-Gault method and actual body weight) were enrolled in the study. Female subjects of childbearing potential had to have a negative serum pregnancy test. Screening laboratory evaluations (hematology, chemistry, and urinalysis) must have fallen within the normal range of the central laboratory's reference ranges, unless the results were determined by the investigator to have no clinical significance. Subjects also had to have either a normal 12-lead electrocardiogram (ECG) or one with abnormalities that were considered clinically insignificant by the investigator in consultation with the sponsor. Subjects who had a history of syncope, palpitations, or unexplained dizziness; or who had an implanted defibrillator or pacemaker were excluded. Subjects were excluded if they had any serious or active medical or psychiatric illness or were receiving any prescription medications or over the counter medications, including herbal products, within 28 days of commencing study drug dosing, with the exception of vitamins, acetaminophen, ibuprofen, and/or hormonal contraceptive medications. Additionally, subjects who had been treated with systemic steroids, immunosuppressant therapies, or chemotherapeutic agents within 3 months of study screening were excluded. Subjects with a history of liver disease, including Gilbert's Disease, were excluded. Subjects with current alcohol or substance abuse were also excluded. Additional restrictions while participating in the study included nicotine-containing products, grapefruit juice or grapefruit, and beverages containing caffeine and other methylxanthines (only on dosing days).

All subjects provided written informed consent before participating in the study. The protocol was reviewed and approved by the Independent Investigational Review Board, Inc. (Plantation, FL), and the study was performed in accordance with the principles of good clinical practice and the Declaration of Helsinki, consistent with the requirements of the US Code of Federal Regulations (CFR) Title 21, Part 312.

\section{Study protocol}

This was a randomized, single-dose, open-label, 3-way crossover, Phase 1 study. Two distinct formulations of the fixed-dose single-tablet regimen containing $200 \mathrm{mg}$ of emtricitabine, $25 \mathrm{mg}$ of rilpivirine, and $300 \mathrm{mg}$ of tenofovir disoproxil fumarate (FTC/RPV/TDF; test formulation [Test Formulations 1 and 2]) were compared with concurrent administration of the individual components (emtricitabine 200-mg capsule, rilpivirine 25-mg tablet, and tenofovir disoproxil fumarate 300 -mg tablet; reference formulation) to healthy adults under fed conditions (standardized meals of $\sim 400 \mathrm{kcal}$ and $\sim 13$ grams of fat). The duration of the study was 43 days and included 3 dosing periods. Period 1 and 2 doses were followed by a 14-day washout. Following period 3 dosing, subjects returned to the study center 14 days after the last dose for a follow-up visit.

Subjects were randomized to 1 of 6 treatment sequences. Following an overnight fast ( $\geq 8$ hours), a single dose of study drug(s) was administered on Days 1, 15, and 29 within 5 minutes of completing a standardized meal of $\sim 400 \mathrm{kcal}$. Following study treatment administration, subjects were restricted from food intake until after the 4-hour pharmacokinetic (PK) blood sampling time point. Mouth checks were performed after each study drug administration to ensure that doses were ingested.

Serial blood samples for PK assessments were collected at the following time points: 0 (predose), $0.25,0.5,0.75,1,1.5,2,2.5,3,3.5$, $4,4.5,5,5.5,6,8,10,12,18,24,36,48,72,96,120,144,168$, and 192 hours after administration of each treatment. Plasma PK sampling was conducted over 8 days after each treatment to completely characterize the PK profile of all 3 agents, especially rilpivirine, which exhibits a long terminal half-life of $\sim 50$ hours [7]. A washout of 14 days between treatments was also provided. Pharmacokinetic results for the coformulation carried forward to commercialization (Test Formulation 1) are presented in this paper.

\section{Safety assessments}

Safety was evaluated by clinical laboratory assessments, ECGs, physical examinations, and vital signs at various time points during the study. Adverse events (AEs) and use of concomitant medications were documented throughout the duration of the study. Treatmentemergent AEs associated with a treatment were defined as events that began on or after the date of the first dose of that treatment and on or before the date of the last dose of that treatment plus 30 days, or before the start date of the following treatment (if applicable), whichever came earlier. Adverse events were coded using the Medical Dictionary for Regulatory Activities (MedDRA), version 12.1. The severity of AEs and laboratory abnormalities was graded according to the Gilead Sciences Modified National Institute of Allergy and Infectious Diseases (NIAID) Common Toxicity Grading Scale $($ mild $=$ Grade 1 , moderate $=$ Grade 2, severe $=$ Grade 3, and possibly life-threatening $=$ Grade 4) [13]

\section{Bioanalytic procedures}

Concentrations of emtricitabine, rilpivirine, and tenofovir in human plasma samples were determined using fully validated highperformance liquid chromatography-tandem mass spectroscopy (LC/MS/MS) bioanalytical methods. Briefly, the methodology for emtricitabine, rilpivirine, and tenofovir was as follows: $100 \mu \mathrm{L}$ of human plasma was deproteinized using methanol solution spiked with internal standard (IS). After protein precipitation extraction, emtricitabine (IS: $\left[{ }^{13} \mathrm{C},{ }^{15} \mathrm{~N}_{2}\right]$-FTC), rilpivirine (IS: $\left[\mathrm{d}_{4},{ }^{13} \mathrm{C}\right]-\mathrm{RPV}$ ), and tenofovir (IS: $T F V-\mathrm{d}_{6}$ ) were resolved on a reverse-phase chromatographic system under isocratic conditions, with a lower limit of quantitation of $5 \mathrm{ng} / \mathrm{mL}$ for emtricitabine and tenofovir and $1 \mathrm{ng} /$ $\mathrm{mL}$ for rilpivirine. Emtricitabine, rilpivirine, and tenofovir and their internal standards were detected in the selected reaction monitoring mode using electrospray ionization (ESI) with positive polarity and the following ion transitions: $\mathrm{m} / \mathrm{z} 248 \rightarrow \mathrm{m} / \mathrm{z} 130$ for emtricitabine, $\mathrm{m} / \mathrm{z} 367 \rightarrow \mathrm{m} / \mathrm{z} 224$ for rilpivirine, $\mathrm{m} / \mathrm{z} 288 \rightarrow \mathrm{m} / \mathrm{z} 176$ for tenofovir, $\mathrm{m} / \mathrm{z} \quad 251 \rightarrow \mathrm{m} / \mathrm{z} 133$ for $\left[{ }^{13} \mathrm{C}^{15} \mathrm{~N}_{2}\right]$-FTC, $\mathrm{m} / \mathrm{z} \quad 372 \rightarrow \mathrm{m} / \mathrm{z} 225$ for $\left[\mathrm{d}_{4^{\prime}}\right.$ $\left.{ }^{13} \mathrm{C}\right]-\mathrm{RPV}$, and $\mathrm{m} / \mathrm{z} 294 \rightarrow \mathrm{m} / \mathrm{z} 182$ for TFV- $\mathrm{d}_{6}$. Calibration curves for 
emtricitabine, rilpivirine, and tenofovir were obtained using a linear regression algorithm of the peak area ratio of each analyte to the IS versus concentration.

The precision and accuracy in the assay validation were evaluated using 3 separate analytic runs, with each containing quality control (QC) samples $(\mathrm{n}=4-5)$ in replicates of 6 for emtricitabine, rilpivirine, and tenofovir. Interassay and intra-assay precision had a $<9.5 \%$ coefficient of variation, and accuracy was within $10 \%$ of expected.

\section{Pharmacokinetic analysis}

The PK analysis sets for emtricitabine, rilpivirine, and tenofovir included all randomized and treated subjects who had respective, evaluable emtricitabine, rilpivirine, and tenofovir PK profiles for the reference formulation and for at least 1 of the 2 test formulations. All predose sample times were assigned a value of 0 . Samples that were below the limit of quantitation (BLQ) of the bioanalytical assay and that were measured before the first quantifiable concentration was achieved were assigned a concentration of 0 to prevent overestimation of the initial area under the plasma concentration-time curve (AUC). Samples that were BLQ at all other time points were treated as missing data.

Pharmacokinetic parameters were estimated (by application of a nonlinear model using standard noncompartmental methods [WinNonlin ${ }^{\circledR}$, version 5.2; Pharsight Corporation, Mountain View, $\mathrm{CA}]$ ) from the plasma concentration-time data for the 3 analytes (emtricitabine, rilpivirine, and tenofovir) after administration of single dose(s) of test and reference formulation. The following PK parameters were estimated: maximum observed plasma concentration of drug $\left(\mathrm{C}_{\max }\right)$, time to maximum concentration $\left(\mathrm{T}_{\max }\right)$, last observed quantifiable concentration of the drug in plasma $\left(\mathrm{C}_{\text {last }}\right)$, observed time point of $\mathrm{C}_{\text {last }}\left(\mathrm{T}_{\text {last }}\right)$, terminal elimination rate constant $\left(\lambda_{\mathrm{z}}\right)$, area under the plasma concentration versus time curve from time 0 to the last quantifiable concentration $\left(\mathrm{AUC}_{0-\text { last }}\right)$, area under the plasma concentration versus time curve from time 0 extrapolated to infinity $\left(\mathrm{AUC}_{\text {inf }}\right)$, \%AUCexp, and terminal elimination half-life $\left(\mathrm{T}_{1 / 2}\right)$.

\section{Statistical analysis}

Statistical power calculations to reject the null hypothesis that there is at least a $20 \%$ difference in each of these $3 \mathrm{PK}$ parameters between each of the 2 test formulations (Test Formulations 1 and 2) and reference formulation were performed using a SAS ${ }^{\circledast}$ (SAS Institute, Cary, NC) macro. This macro was developed on the basis of the method proposed by Chow and Liu [14] for William Design assuming no unbalanced carry-over effects and each of two 1-sided tests to be performed at an alpha level of 5\%. Preliminary data from a previous study (emtricitabine, rilpivirine, and tenofovir unpublished data on file at Gilead Sciences) were used to estimate the intrasubject standard deviations (SDs) for PK parameters $\left(\mathrm{AUC}_{\text {inf }}, \mathrm{AUC}_{0 \text {-last }}\right.$, and $\mathrm{C}_{\max }$ ) for emtricitabine, rilpivirine, and tenofovir. Power analyses estimated that 24 evaluable subjects would provide at least a $99 \%$ probability that bioequivalence was supported if the expected geometric mean ratio (test/reference) was 1.00 for each analyte in terms of the primary endpoints $\mathrm{AUC}_{\text {inf }}, \mathrm{AUC}_{0-\text { last }}$, and $\mathrm{C}_{\max }$, and at least a $97 \%$ probability that bioequivalence would be supported if the expected geometric mean ratio (test/reference) was 1.05 for each PK parameter of each analyte. A sample size of 24 subjects also would provide at least a $95 \%$ $(99 \% \times 97 \% \times 99 \%)$ aggregated power to conclude bioequivalence for all 3 analytes simultaneously (i.e., to determine overall bioequivalence between the test and reference formulations). Given the long duration of the study, a larger overage (50\%) was built into the study sample size to account for dropouts, thus requiring a total enrollment of 36 subjects (6 per treatment sequence).

Subject demographic data, baseline characteristics, and PK parameters were summarized by treatment using descriptive statistics. To evaluate the bioequivalence of each test formulation (1 and 2) versus the reference formulation, natural log-transformed primary $\mathrm{PK}$ values $\left(\mathrm{AUC}_{\text {inf }}, \mathrm{AUC}_{0 \text {-last }}\right.$, and $\mathrm{C}_{\max }$ ) for emtricitabine, rilpivirine, and tenofovir were compared between the test and reference formulations by a parametric analysis of variance (ANOVA) using SAS ${ }^{\circledR}$ PROC MIXED (SAS Institute, Cary, NC) appropriate for the 3-way crossover design of the study.

Rilpivirine exhibits a long plasma half-life (median $\mathrm{T}_{1 / 2} \sim 50$ hours) [7] that could have resulted in detectable drug levels being observed at earlier time points in subjects during dosing periods 2 and 3 . A complex model (referred to as "full model") including treatment, sequence, period, and first order of carry-over effects as fixed effects, and subject within sequence as a random effect was used to test whether there were unequal carry-over effects for rilpivirine. No statistically significant unequal carry-over effects were detected and a reduced model without carry-over effects was used for all PK parameters of emtricitabine, rilpivirine, and tenofovir. Treatment comparisons and $90 \%$ confidence intervals (CIs) were constructed using the reduced model. Ninety percent CIs for the ratio of the geometric least-squares means (test formulation/reference formulation) were calculated for each parameter, consistent with the two 1 -sided tests approach $[15,16]$. Per scientific standards for bioequivalence studies, formulation bioequivalence was concluded if the $90 \%$ CI for the ratio of the geometric least-squares means was contained within the bounds of $80 \%$ to $125 \%$ for the $\mathrm{AUC}_{\text {inf }}$ $\mathrm{AUC}_{0 \text {-last }}$, and $\mathrm{C}_{\max }$ values of all 3 analytes (emtricitabine, rilpivirine, and tenofovir) $[15,17]$.

\section{Results}

\section{Subject demographics and disposition}

A total of 36 healthy subjects were enrolled in the study and received at least 1 dose of study drug (test [formulation 1 or 2] or reference formulation). Of the 36 enrolled subjects, 15 (41.7\%) were male, 27 (75.0\%) were white, and 9 (25.0\%) were black. The mean (SD) age was 33 (7.0) years, mean (SD) weight at screening was 71.9 (10.97) kg, mean (SD) height at screening was $167.9(9.68) \mathrm{cm}$, mean BMI at screening was $25.4(2.48) \mathrm{kg} / \mathrm{m}^{2}$, and the mean (SD) estimated creatinine clearance by Cockcroft-Gault method at baseline was 128.0 (17.91) $\mathrm{mL} /$ minute. Thirty-four subjects completed the study; 1 subject received only the fixed-dose FTC/RPV/TDF Test Formulation 2 (noncommercialized formulation) and discontinued due to an $\mathrm{AE}$ of angioedema of the face, and 1 subject received only the reference formulation and was discontinued by the investigator for a protocol violation (positive drug test).

\section{Safety}

Single oral doses of emtricitabine, rilpivirine, and tenofovir disoproxil fumarate administered as individual drugs given concurrently or as a fixed-dose combination tablet were generally well tolerated in this study. Treatment-emergent AEs were reported in 3 of 35 subjects (8.6\%) after administration of the reference formulation, 4 of 34 subjects (11.8\%) after administration of the fixed-dose FTC/RPV/ TDF Test Formulation 1 (bioequivalent commercial formulation), and 4 of 35 subjects (11.4\%) after administration of the fixed-dose FTC/ 
RPV/TDF Test Formulation 2. Drug-related treatment-emergent AEs were reported for 2 subjects from each treatment group, and included diarrhea, musculoskeletal pain, pain in extremity, headache, dysuria, testicular pain, angioedema, papular rash, and hot flash. The drug-related $\mathrm{AE}$ of Grade 1 angioedema of the right side of the face following administration of the first dose of study treatment (FTC/ RPV/TDF Test Formulation 2) led to discontinuation of the subject from the study. Most AEs were mild, transient, and consistent with the known emtricitabine, rilpivirine, and tenofovir disoproxil fumarate safety profiles $[7,18,19]$. No death or serious adverse event occurred during the study.

Fasting hypercholesterolemia (all Grade 1 events) was the most frequent treatment-emergent graded laboratory abnormality and was reported in 6 of 34 subjects (17.6\%) after administration of the reference formulation, 1 of 34 subjects (2.9\%) after administration of Test Formulation 1, and 2 of 35 subjects (5.7\%) after administration of Test Formulation 2. No notable change in clinical laboratory test value treatment means, vital signs, or physical findings was observed during the study. No pregnancy occurred during the study.

\section{Pharmacokinetics}

The PK analyses data sets for emtricitabine, rilpivirine, and tenofovir each included 34 subjects. Two subjects who prematurely discontinued from the study (discussed above) were excluded from the emtricitabine, rilpivirine, and tenofovir PK analysis sets. Mean (SD) emtricitabine, rilpivirine, and tenofovir plasma concentrationtime profiles are presented in Figure 1 (A to C, respectively). The plasma concentrations of emtricitabine, rilpivirine, and tenofovir were similar after administration of the fixed-dose combination Test Formulation 1 or concurrent administration of the individual drugs (reference formulation) during the monitoring period (up to 192 hours after dosing). Derived plasma PK parameters for emtricitabine, rilpivirine, and tenofovir after administration of the reference or test formulation are presented in Table 1 , Table 2 , and Table 3 , respectively. The FTC/RPV/TDF fixed-dose combination Test Formulation 2 was not bioequivalent to the reference formulation products as not all 3 of the components of the fixed-dose formulation, specifically RPV C met the bioequivalence criterion. Accordingly, only results from Test Formulation 1 taken to commercialization are presented in this paper.

The FTC/RPV/TDF single-tablet regimen (Test Formulation 1) demonstrated bioequivalence to the reference formulation of coadministration of the individual components (emtricitabine + rilpivirine + tenofovir disoproxil fumarate) under fed conditions. The $90 \%$ CIs for the ratio of the geometric least-squares means of the primary $\mathrm{PK}$ parameters $\left(\mathrm{AUC}_{\mathrm{inf}}, \mathrm{AUC}_{0 \text {-last }}\right.$, and $\left.\mathrm{C}_{\max }\right)$ for test formulation 1 versus the reference formulation were contained within the bounds of $80 \%$ to $125 \%$ for emtricitabine, rilpivirine, and tenofovir. The coformulated FTC/RPV/TDF single-tablet regimen test formulation 1 and coadministered emtricitabine + rilpivirine + tenofovir disoproxil fumarate also exhibited similar median $\mathrm{T}_{1 / 2}$ values. Statistical comparisons of emtricitabine, rilpivirine, and tenofovir PK parameters after administration of test formulation or reference formulation are summarized in Table 4.

\section{Discussion}

Current guidelines for the treatment of ARV treatment-naïve HIV-1 infected patients recommend that initial therapy be comprised of 2 NRTIs/NtRTIs and either an NNRTI (efavirenz or nevirapine),
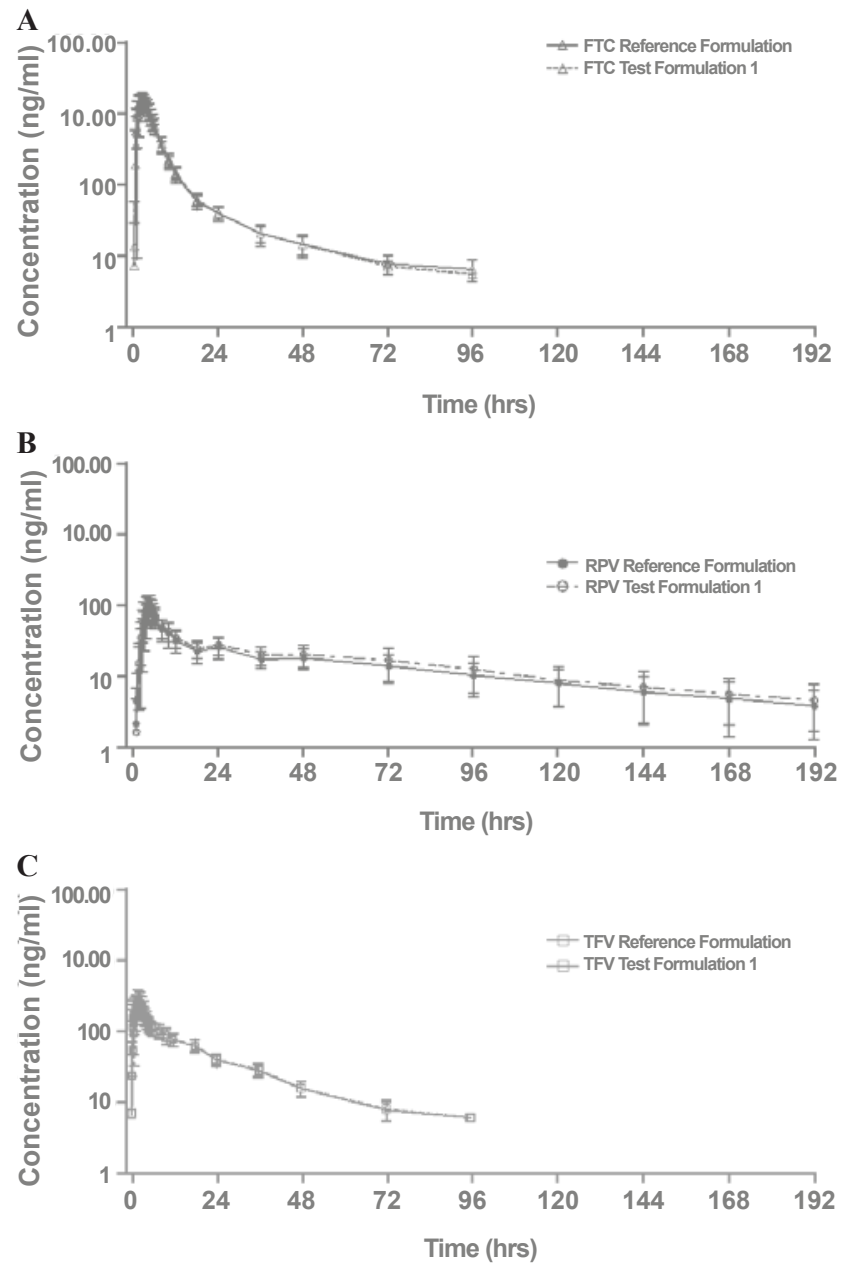

Mean $(S D)$ plasma concentration-time profiles $(n=34)$ of emtricitabine $(A)$, rilpivirine (B) and tenofovir (C) over 192 hours after administration of Test Formulation 1 and Reference formulation. Measurable concentrations of emtricitabine and tenofovir ( $A$ and $C$, respectively) were observed up to 96 hours after administration of study treatments.

FTC = emtricitabine; RPV = rilpivirine; TFV = tenofovir

Figure 1: Emtricitabine, Rilpivirine, and Tenofovir Pharmacokinetics $(n=34)$.

\begin{tabular}{|c|c|c|}
\hline Emtricitabine PK Parameter & $\begin{array}{l}\text { Test } \\
\text { Formulation } 1 \\
(\mathrm{n}=34)^{\mathrm{a}}\end{array}$ & $\begin{array}{l}\text { Reference } \\
\text { Formulation } \\
(\mathrm{n}=34)^{\mathrm{a}}\end{array}$ \\
\hline $\mathrm{C}_{\max }(\mathrm{ng} / \mathrm{mL})$, mean $(\% \mathrm{CV})$ & $1750(23.6)$ & $1650(21.9)$ \\
\hline $\mathrm{T}_{\max }$ (hours), median (Q1, Q3) & $2.50(2.00,3.00)$ & $2.00(2.00,2.50)$ \\
\hline $\mathrm{C}_{\text {last }}(\mathrm{ng} / \mathrm{mL})$, mean $(\% \mathrm{CV})$ & $8.6(35.0)$ & $8.2(29.1)$ \\
\hline $\mathrm{T}_{\text {last }}$ (hours), median (Q1, Q3) & $72.0(48.0,72.0)$ & $72.0(48.0,72.0)$ \\
\hline $\mathrm{AUC}_{0 \text {-last }}(\mathrm{ng} \cdot \mathrm{h} / \mathrm{mL})$, mean $(\% \mathrm{CV})$ & 9420 (14.3) & $9420(13.9)$ \\
\hline $\mathrm{AUC}_{\text {inf }}(\mathrm{ng} \cdot \mathrm{h} / \mathrm{mL})$, mean $(\% \mathrm{CV})$ & 9640 (14.1) & $9640(13.6)$ \\
\hline$\% \mathrm{AUC}_{\text {exp }}$, mean $(\% \mathrm{CV})$ & $2.3(45.4)$ & $2.4(49.7)$ \\
\hline $\mathrm{T}_{1 / 2}$ (hours), median (Q1, Q3) & $18.3(14.3,20.2)$ & $19.0(14.4,20.8)$ \\
\hline
\end{tabular}

$\% \mathrm{CV}=\%$ coefficient of variation; $\mathrm{PK}=$ pharmacokinetic; $\mathrm{Q} 1=$ first interquartile; $\mathrm{Q} 3$ $=$ third interquartile

Data presented in 3 significant figures.

a: Two subjects were excluded from the PK analysis set due to premature discontinuation from the study.

Table 1: Summary of Emtricitabine Pharmacokinetic Parameters.

a boosted protease inhibitor (PI), or the INSTI raltegravir [3-6]. The recent approval of an alternative NNRTI, rilpivirine, by the US FDA as an once-daily oral treatment for HIV-1 infection in combination 


\begin{tabular}{|c|c|c|}
\hline Rilpivirine PK Parameter & $\begin{array}{l}\text { Test } \\
\text { Formulation } 1 \\
(\mathrm{n}=34)^{\mathrm{a}}\end{array}$ & $\begin{array}{l}\text { Reference } \\
\text { Formulation } \\
(\mathrm{n}=34)^{\mathrm{a}}\end{array}$ \\
\hline $\mathrm{C}_{\max }(\mathrm{ng} / \mathrm{mL})$, mean $(\% \mathrm{CV})$ & $116(29.6)$ & $99.8(30.5)$ \\
\hline $\mathrm{T}_{\text {max }}$ (hours), median (Q1, Q3) & $4.50(4.00,4.50)$ & $4.50(4.00,4.50)$ \\
\hline $\mathrm{C}_{\text {last }}(\mathrm{ng} / \mathrm{mL})$, mean $(\% \mathrm{CV})$ & $4.1(72.9)$ & $3.4(72.8)$ \\
\hline $\mathrm{T}_{\text {last }}$ (hours), median (Q1, Q3) & $192(192,193)$ & $192(192,192)$ \\
\hline $\mathrm{AUC}_{0 \text {-last }}(\mathrm{ng} \cdot \mathrm{h} / \mathrm{mL})$, mean $(\% \mathrm{CV})$ & $3010(34.5)$ & $2600(32.5)$ \\
\hline$A \cup C_{\text {inf }}(\mathrm{ng} \cdot \mathrm{h} / \mathrm{mL})$, mean $(\% \mathrm{CV})$ & $3390(39.4)$ & $2920(38.6)$ \\
\hline$\% A \cup C_{\text {exp }}$, mean $(\% \mathrm{CV})$ & $9.5(68.5)$ & $9.6(69.3)$ \\
\hline $\mathrm{T}_{1 / 2}$ (hours), median (Q1, Q3) & $54.2(39.0,62.9)$ & $53.1(40.2,65.5)$ \\
\hline
\end{tabular}

Table 2: Summary of Rilpivirine Pharmacokinetic Parameters.

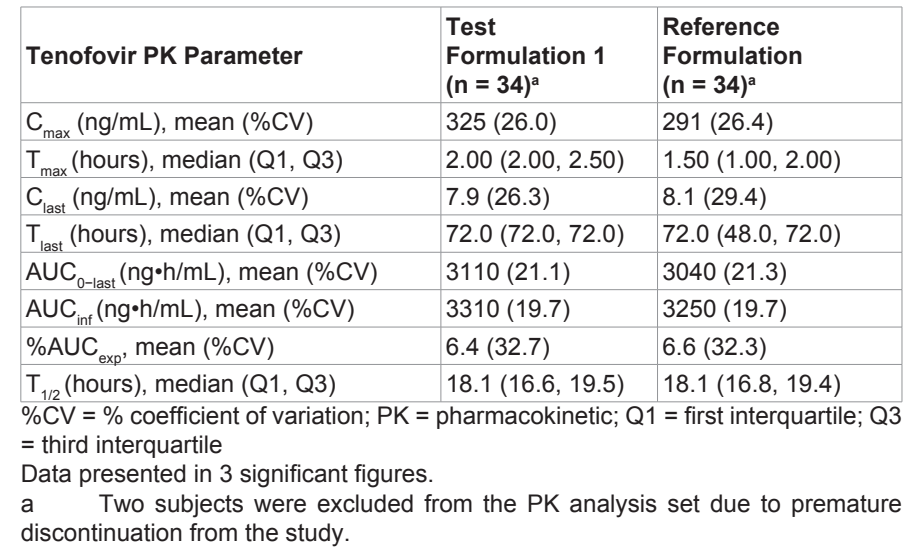

Table 3: Summary of Tenofovir Pharmacokinetic Parameters.

\begin{tabular}{|c|c|c|c|c|c|c|}
\hline \multirow[b]{2}{*}{ PK Parameter } & \multicolumn{2}{|l|}{ Emtricitabine } & \multicolumn{2}{|l|}{ Rilpivirine } & \multicolumn{2}{|l|}{ Tenofovir } \\
\hline & $\begin{array}{l}\text { Geometric } \\
\text { Least-Squares } \\
\text { Mean Ratio (\%) }\end{array}$ & $90 \% \mathrm{Cl}$ & $\begin{array}{l}\text { Geometric } \\
\text { Least-Squares Mean } \\
\text { Ratio (\%) }\end{array}$ & $90 \% \mathrm{Cl}$ & $\begin{array}{l}\text { Geometric } \\
\text { Least-Squares Mean Ratio (\%) }\end{array}$ & $90 \% \mathrm{Cl}$ \\
\hline $\mathrm{C}_{\max }$ & 105 & 100 to 111 & 116 & 108 to 124 & 111 & 104 to 118 \\
\hline$A \cup C_{0 \text {-last }}$ & 99.9 & 97.8 to 102 & 116 & 109 to 123 & 102 & 99.0 to 105 \\
\hline$A \cup C_{\text {inf }}$ & 99.9 & 97.7 to 102 & 116 & 109 to 123 & 102 & 99.1 to 105 \\
\hline
\end{tabular}

$\mathrm{Cl}=$ confidence interval; $\mathrm{PK}=$ pharmacokinetic

Data presented in 3 significant figures

Table 4: Statistical Comparisons of Emtricitabine, Rilpivirine, and Tenofovir Pharmacokinetic Parameters for Test Formulation 1 versus Reference Formulation.

with other ARV agents in ARV treatment-naïve adults may offer an attractive treatment option to patients wishing to avoid NNRTI regimens containing efavirenz or nevirapine due to tolerability concerns and/or potential reproductive risks. Efavirenz has been associated with increases in serum triglycerides and low-density lipoprotein (LDL), neurological, psychological, and dermatological side effects, and is potentially teratogenic. Hepatotoxicity and severe rash/hypersensitivity reactions have been associated with the use of nevirapine.

The present study confirmed that the commercial formulation of the FTC/RPV/TDF single-tablet regimen is bioequivalent to the concurrent administration of the individual components under fed conditions (standardized meal $\sim 400 \mathrm{kcal}$ ). Absorption of rilpivirine has been shown to be reduced by $40 \%$ when administered in fasting conditions compared to administration following a normal or high-caloric meal [7]. Therefore, it is recommended that FTC/RPV/ TDF single-tablet regimen be administered with a meal [12]. The complementary long intracellular (emtricitabine $\sim 39$ hours $[20,21]$ and tenofovir $\sim>60$ hours $[19,20,22]$ ) and plasma (rilpivirine $\sim 50$ hours [12]) half-lives of each of the individual agents in this single-tablet regimen allow for once daily dosing. The simplified once-daily dosing of a single-tablet regimen may be beneficial in maximizing patient adherence in order to improve clinical outcomes.

Single oral doses of emtricitabine, rilpivirine, and tenofovir disoproxil fumarate administered as individual drugs given concurrently or as a fixed-dose single-tablet regimen were generally well tolerated by the subjects in this study. Drug-related treatment-emergent AEs included diarrhea, musculoskeletal pain, pain in extremity, headache, dysuria, testicular pain, angioedema, papular rash, and hot flash. Most AEs reported were mild, transient, and consistent with the known emtricitabine, rilpivirine, and tenofovir disoproxil fumarate safety profiles $[7,18,19]$.

Rilpivirine is classified as a Biopharmaceutics Classification System [BCS] II compound (low solubility/high permeability). These properties in conjunction with the chemical stability of tenofovir disoproxil fumarate presented challenges in developing a co-formulated single tablet. Conventional dry and co-blend granulation methodologies were unsuccessful in developing a bioequivalent single tablet regimen (data on file). Formulation and process modifications were implemented, including bilayer tableting to minimize potential physical and chemical interactions between emtricitabine, rilpivirine, and tenofovir disoproxil fumarate. The test formulation 1 of the FTC/RPV/TDF single-tablet regimen found to be bioequivalent to the coadministration of the individual agents in this study is a bilayer tablet, a design that facilitates tablet disintegration and dissolution. This formulation was approved for commercialization by the US FDA in August 2011 and in the EU in November 2011, and is currently undergoing regulatory review in other countries.

In conclusion, this study demonstrated that the commercial formulation of the FTC/RPV/TDF single-tablet regimen is bioequivalent to the concurrent administration of the individual components under fed conditions (standardized meal $\sim 400 \mathrm{kcal}$ ). The FTC/RPV/TDF single-tablet regimen is a next-generation, once-daily single-tablet complete ARV regimen for the treatment of HIV-1 infection.

\section{Acknowledgments}

We thank the healthy volunteers for their participation. We also acknowledge Jennifer DeMorin and Susan Chuck (Gilead Sciences) for their key reviewer contributions. Medical writing and editing services were provided by Sage Scientific Writing, LLC. The managing members of Sage Scientific Writing, LLC are former employees of Gilead Sciences, Inc

\section{References}

1. Airoldi M, Zaccarelli M, Bisi L, Bini T, Antinori A, et al. (2010) One-pill once-a- 
Citation: Mathias A, Menning M, Wiser L, Wei X, Dave A, et al. (2012) Bioequivalence of the Emtricitabine/Rilpivirine/Tenofovir Disoproxil Fumarate Single Tablet Regimen. J Bioequiv Availab 4: 100-0105. doi:10.4172/jbb.1000121

day HAART: a simplification strategy that improves adherence and quality of life of HIV-infected subjects. Patient Prefer Adherence 4: 115-125

2. Kitahata MM, Reed SD, Dillingham PW, Van Rompaey SE, Young AA, et al (2004) Pharmacy-based assessment of adherence to HAART predicts virologic and immunologic treatment response and clinical progression to AIDS and death. Int J STD AIDS 15: 803-810.

3. US Department of Health and Human Services (2011) Panel on Antiretroviral Guidelines for Adults and Adolescents. Guidelines for the use of antiretroviral agents in HIV-1-infected adults and adolescents. Department of Health and Human Services. 1-167.

4. European AIDS Clinical Society (EACS) (2011) Guidelines for the Clinica Management and Treatment of HIV Infected Adults in Europe. Version 6.0: $1-61$.

5. Thompson MA, Aberg JA, Cahn P, Montaner JS, Rizzardini G, et al. (2010) Antiretroviral treatment of adult HIV infection: 2010 recommendations of the International AIDS Society-USA panel. JAMA 304: 321-333.

6. Gazzard BG, Anderson J, Babiker A, Boffito M, Brook G, et al. (2008) British HIV Association Guidelines for the treatment of HIV-1-infected adults with antiretroviral therapy 2008. HIV Med 9: 563-608.

7. Edurant (2011). Raritan, NJ: Tibotec Pharmaceuticals.

8. Pozniak AL, Morales-Ramirez J, Katabira E, Steyn D, Lupo SH, et al. (2010) Efficacy and safety of TMC278 in antiretroviral-naive HIV-1 patients: week 96 results of a phase Ilb randomized trial. AIDS 24: 55-65.

9. Molina JM, Cahn P, Grinsztejn B, Lazzarin A, Mills A, et al. (2011) Rilpivirine versus efavirenz with tenofovir and emtricitabine in treatment-naive adults infected with HIV-1 (ECHO): a phase 3 randomised double-blind activecontrolled trial. Lancet 378: 238-246.

10. Cohen CJ, Andrade-Villanueva J, Clotet B, Fourie J, Johnson MA, et al. (2011) Rilpivirine versus efavirenz with two background nucleoside or nucleotide reverse transcriptase inhibitors in treatment-naive adults infected with HIV-1 (THRIVE): a phase 3, randomised, non-inferiority trial. Lancet 378: 229-237.

11. Desmidt M, Willems B, Dom P, Bailey G, De Schaepdrijver L, et al. (2009) Absence of a teratogenic potential from a novel next generation NNRTI,
TMC278. Poster presented at: 12th European AIDS Conference. Cologne Germany.

12. Complera (2011) Foster City, CA: Gilead Sciences, Inc

13. Regulatory Compliance Center (1992) Division of AIDS (DAIDS) Table of Grading Severity of Adult Adverse Experiences. Bethesda, MD, National Institute of Allergy and Infectious Diseases.

14. Chow SC, Liu JP (2008) Assessment of Bioequivalence for More Than Two Formulations. In Chow SC, Liu JP, eds. Design and Analysis of Bioavailability and Bioequivalence Studies. 3rd ed. Boca Raton, FL: CRC Press. 301-328.

15. US Department of Health and Human Services Food and Drug Administration Center for Drug Evaluation and Research (CDER) (2003) Guidance for Industry. Bioavailability and Bioequivalence Studies for Orally Administered Drug Products-General Considerations.

16. U.S. Department of Health and Human Services Food and Drug Administration Center for Drug Evaluation and Research (CDER) (2001) Guidance for Industry. Statistical Approaches to Establishing Bioequivalence.

17. European Medicines Agency (EMEA). Committee for Medicinal Products fo Human Use (CHMP) (2010) Guideline on the investigation of bioequivalence.

18. Emtriva (2008) Foster City, CA: Gilead Sciences, Inc

19. Kulkarni R, Babaoglu K, Lansdon EB, Rimsky L, Van Eygen V, et al. (2012) The HIV-1 reverse transcriptase M184I mutation enhances the E138K-associated resistance to rilpivirine and decreases viral fitness. J Acquir Immune Defic Syndr 59: 47-54.

20. Mathias AA, Hinkle J, Menning M, Hui J, Kaul S, et al. (2007) Bioequivalence of efavirenz/emtricitabine/tenofovir disoproxil fumarate single-tablet regimen. J Acquir Immune Defic Syndr 46: 167-173.

21. Molina JM, Cox SL (2005) Emtricitabine: a novel nucleoside reverse transcriptase inhibitor. Drugs Today (Barc) 41: 241-252.

22. Hawkins T, Veikley W, St Claire RL $3^{\text {rd }}$, Guyer B, Clark N, et al. (2005) Intracellular pharmacokinetics of tenofovir diphosphate, carbovir triphosphate and lamivudine triphosphate in patients receiving triple-nucleoside regimens. J Acquir Immune Defic Syndr 39: 406-411. 